\title{
Preface to the Special Issue on Language-Based Interaction
}

\author{
SANDRA CARBERRY and INGRID ZUKERMAN
}

Natural Language (NL) has been linked with User Modeling since the inception of the User Modeling field. In fact, the first User Modeling workshop, which was held in Maria Laach, Germany, in 1986, focused on the application of User Modeling to dialogue systems (Kobsa and Wahlster, 1989). These applications were primarily motivated by the insight that understanding people's utterances involves building and consulting a model of the interlocutor, and generating felicitous discourse requires taking into account a model of the addressee. Thus, the applications of the time focused on three main language-related aspects of user modeling: (1) building user models from NL utterances, e.g., (Allen and Perrault, 1980; Litman and Allen, 1987; Carberry, 1990), (2) consulting user models to interpret users' utterances (Cohen and Jones, 1989; Carberry, 1990), and (3) consulting user models to generate discourse that takes into account some aspect of the addressee (Jameson, 1989; McCoy, 1989; Paris, 1989). These applications were typically knowledge intensive - they relied on plan libraries and applied rule-based reasoning to infer user models or generate discourse.

Since then, the applications in the field of User Modeling have expanded significantly. User Modeling now includes new applications, such as recommender systems, e-commerce, mobile and ubiquitous systems, personalized TV, and group modeling. This expansion has coincided with a methodological shift in the Artificial Intelligence community towards techniques which have a strong probabilistic or empirical basis, such as Bayesian networks and machine learning techniques. This shift in turn has been accompanied by a requirement for a rigorous evaluation of developed systems.

The articles in this Special Issue reflect this trend with respect to systems that address the three main aspects at the intersection of User Modeling and NL. Goodman et al. use machine learning techniques in a system that builds models of user interactions, and Komatani et al. apply these techniques to build models of users of a spoken dialogue system. Michaud et al. also build user models, but with a rule-based approach, while Bontcheva and Wilks apply rules for NL generation. Finally, Zukerman and George employ probabilistic methods in a discourse interpretation system that consults a user model. 
Goodman et al. utilize a variety of machine learning methodologies (CART models, Hidden Markov Models, and neural networks) that analyze features of language interaction during a collaborative learning experience, and build models of the individuals and the group. These models are used by an intelligent peer agent who oversees the learning activity and intervenes to facilitate a successful learning experience for all the participants. The individual components of the system were evaluated using collected dialogues from students engaged in a collaborative design task.

The spoken language system of Komatani et al. produces responses that are tailored to an individual's skill level in using the system, their knowledge of the target domain, and the urgency with which they require information. These aspects, which are learned by applying decision trees to the features of users' utterances, influence the length of a response, its specific domain content, and the inclusion of dialogue management information. The system was evaluated in the context of a city bus information provider.

The system described by Bontcheva and Wilks tailors the content and structure of automatically generated hypertext to a user's beliefs and preferences. An important aspect of this system is its reliance on a reusable user-modeling component, which is consulted to adapt the hypertext content and links. The user-modeling and adaptive features of the system were evaluated by means of a web-based information-gathering task.

Michaud et al. offer a hybrid user model that combines an overlay model with a stereotypical reasoning model. The overlay model captures a user's observed skills, and incomplete information in this model is supplemented by postulating skills based on typical learning sequences in the domain. This hybrid model was implemented and evaluated in an instruction system for users writing in a second language, where the task was to determine the most likely parse of users' sentences.

Finally, Zukerman and George present a probabilistic approach for the interpretation of users' arguments, which is based on the tenet that the intended interpretation is that with the highest posterior probability. They offer a technique for calculating the posterior probability of candidate interpretations, and investigate the incorporation of three types of user models into their formalism. This work was implemented and evaluated in a web-based system where users investigate a murder mystery and argue about it.

As the papers in this special issue demonstrate, User Modeling in Natural Language interaction has made substantial progress since its infancy in the 1980s. These papers point the way forward in different ways - by considering realistic application domains, applying probabilistic and machine learning techniques, and incorporating web-based technologies. One of the main challenges envisaged for the future is the development of systems that combine these aspects and interact with a wide variety of users in open-ended situations. Further, as new applications arise, and as the successes and limitations of current methodologies are doc- 
umented, we expect the field to continue to evolve and address new challenges and opportunities.

\section{References}

Allen, J. F. and Perrault, C. R.: 1980, Analyzing Intention in Utterances. Artificial Intelligence 15, 143-178.

Carberry, S.: 1990, Plan Recognition in Natural Language Dialogue. ACL-MIT Press Series on Natural Language Processing. MIT Press.

Cohen, R. and Jones, M.: 1989, Incorporating User Models into Expert Systems for Educational Diagnosis. In: A. Kobsa and W. Wahlster (eds.): User Models in Dialog Systems. Springer-Verlag, pp. 313-333.

Jameson, A.: 1989, 'But What Will the Listener Think? Belief Ascription and Image Maintenance in Dialog'. In: A. Kobsa and W. Wahlster (eds.): User Models in Dialog Systems. Springer-Verlag, pp. 255-312.

Kobsa, A. and Wahlster, W. (eds.): 1989, User Models in Dialog Systems. Springer-Verlag.

Litman, D. and Allen, J. F.: 1987, A Plan Recognition Model for Subdialogues in Conversation. Cognitive Science 11, 163-200.

McCoy, K. F.: 1989, Generating Context-Sensitive Responses to Object-Related Misconceptions. Artificial Intelligence 41, 157-195.

Paris, C. L.: 1989, The Use of Explicit User Models in a Generation System for Tailoring Answers to the User's Level of Expertise. In: A. Kobsa and W. Wahlster (eds.): User Models in Dialog Systems. Springer-Verlag, pp. 200-232.

\section{Authors' Vitae}

Sandra Carberry is a Professor of Computer Science at the University of Delaware. She received her B.Sc degree in mathematics from Cornell University, her M.Sc. degree in electrical engineering (with a specialization in computer science) from Rice University, and her Ph.D. in computer science from the University of Delaware. Her areas of research are discourse, dialogue, and plan recognition with a current focus on multimodal communication, negotiation, and summarization.

Ingrid Zukerman is an Associate Professor in Computer Science at Monash University. She received her B.Sc. degree in Industrial Engineering and Management and her M.Sc. degree in Operations Research from the Technion - Israel Institute of Technology. She received her Ph.D. degree in Computer Science from UCLA in 1986. Her areas of interest are discourse planning, plan recognition, question answering, and agent modeling. 